\title{
BMJ Open Perspectives on the provision of GDM screening in general practice versus the hospital setting: a qualitative study of providers and patients
}

\author{
Marie Tierney, ${ }^{1}$ Angela O'Dea, ${ }^{1}$ Andrii Danyliv, ${ }^{2}$ Louise Carmody, ${ }^{1}$ \\ Brian E McGuire, ${ }^{3}$ Liam G Glynn, ${ }^{4}$ Fidelma Dunne ${ }^{1}$
}

To cite: Tierney M, O'Dea A, Danyliv A, et al. Perspectives on the provision of GDM screening in general practice versus the hospital setting: a qualitative study of providers and patients. BMJ Open 2016:6:e007949. doi:10.1136/bmjopen-2015007949

- Prepublication history for this paper is available online To view these files please visit the journal online (http://dx.doi.org/10.1136/ bmjopen-2015-007949).

Received 13 February 2015 Revised 13 November 2015 Accepted 26 November 2015

CrossMark

For numbered affiliations see end of article.

Correspondence to Dr Marie Tierney; marie.m.tierney@nuigalway.ie

\section{ABSTRACT}

Objective: A novel gestational diabetes mellitus (GDM) screening programme which involved offering screening at the patient's general practitioner (GP) compared with the traditional hospital setting was trialled. This study investigates perspectives of involved stakeholders on the provision of GDM screening at both settings.

Design: Thematic analysis of the perspectives of stakeholders involved in the receiving and provision of GDM screening in both the GP and hospital settings drawn from focus groups and interviews.

Participants: 3 groups of participants are included in this research - patient participants, GP screening providers and hospital screening providers. All were recruited from a larger sample who participated in a randomised controlled screening trial. Purposeful sampling was utilised to select participants with a wide variety of perspectives on the provision of GDM screening.

Setting: Participants were recruited from a geographical area covered by 3 hospitals in Ireland.

Results: 4 themes emerged from thematic analysisnamely (1) travel distance, (2) best care provision, (3) sense of ease created and (4) optimal screening.

Conclusions: The influence of travel distance from the screening site is the most important factor influencing willingness to attend for GDM screening among women who live a considerable distance from the hospital setting. For patients who live equidistance from both settings, other factors are important; namely the waiting facilities including parking, perceived expertise of screening provider personnel, access to emergency treatment if necessary, accuracy of tests and access to timely results and treatment. Optimal screening for GDM should be specialist led, incorporate expert advice of GDM screening, treatment and management, should be provided locally, offer adequate parking and comfort levels, provide accurate tests, and timely access to results and treatment. Such a service should result in improved rates of GDM screening uptake.

Trial registration number: ISRCTN41202110.

\section{INTRODUCTION}

Gestational diabetes mellitus (GDM) is defined as 'any degree of glucose intolerance

\section{Strengths and limitations of this study}

- The inclusion of all of the key stakeholders involved in screening for gestational diabetes mellitus (GDM) as part of the randomised controlled screening trial is deemed a strength of this study.

- The inclusion of a wide variety of stakeholders' perspectives allows the results to be broadly representative of the general population, another strength of the study.

- A limitation of the study is that the participants of this study were recruited from a defined area along the Irish Atlantic seaboard, potentially limiting the extrapolation of results nationally and internationally.

with onset or first recognition during pregnancy'. ${ }^{1}$ The most recent prevalence estimate for GDM in Ireland is $12.4 \% .^{2}$ GDM prevalence rates are increasing due to increasing maternal age and BMI at pregnancy. ${ }^{3} 4$ Untreated GDM has consequences for both maternal and neonatal health in the short term, including macrosomia, increased risk of caesarean section delivery and increased neonatal unit admission rates. ${ }^{2}{ }^{5-7}$ In addition, a history of GDM is associated with an increased long-term risk for the development of prediabetes and type 2 diabetes mellitus in the mother and her offspring. ${ }^{8-12}$ Screening allows for timely identification of the condition and thus allows for initiation of treatment in a timely and effective manner, where necessary, to minimise or avoid the previously outlined complications.

A study by Gayet-Ageron $e t a l^{13}$ showed that $98 \%$ of women determined that GDM screening was acceptable and $89 \%$ deemed it essential. However, screening uptake rates in Ireland are suboptimal with a most recent estimate of $44 \%$ found by the Atlantic Diabetes in Pregnancy (DIP) consortium, 
when screening was applied universally to a regional pregnant population. ${ }^{2}$ Thus, it is likely that, even in universal screening protocols, a significant proportion of positive cases go undiagnosed. Previous research postulated that distance to the screening site is a barrier to screening uptake and that provision of screening in a location closer to the woman's home would result in improved rates of uptake. ${ }^{14}$

The approach to screening and diagnosis of GDM internationally is disorderly. ${ }^{15}$ The protocols for screening and diagnosis vary not only between countries, but also within countries. In Europe, selective screening based on the presence of specified risk factors is more frequently implemented in comparison with the USA and Canada, where universal screening is the predominant practice. A variety of screening tests are also utilised worldwide including the $2 \mathrm{~h} 75$ or $100 \mathrm{~g}$ oral glucose tolerance test (OGTT), random glucose test, $50 \mathrm{~g}$ glucose challenge test and fasting glucose test. The diagnostic criteria for GDM also varies greatly and includes the 2013 WHO criteria, the 1999 WHO criteria, European Association for the Study of Diabetes (EASD) criteria, Carpenter and Coustan criteria, and various national standards. Less well researched is the variety of locations in which GDM screening occurs. In Europe generally, screening is typically hospital based, whereas the practice of general practitioner (GP)/community screening appears to be practiced on a greater level in countries such as the USA, Canada and New Zealand.

Screening by GPs is successful for screening programmes in Ireland, for example, CervicalCheck-The National Cervical Screening Programme. A randomised controlled trial was undertaken in order to assess if uptake rates for GDM screening improved when offered at the patient's GP setting compared with the hospital setting. ${ }^{16}$ However, in this trial, uptake rates were superior in the hospital setting compared with the GP setting, despite the prior indications of Cullinan et al. ${ }^{14}$

This study sought to understand the perspectives about screening in both the GP and hospital settings from the viewpoints of patients screened in both locations and the GP and hospital providers of the screening in order to provide context to the findings of the randomised controlled screening trial. Understanding stakeholders' perspectives is key to identifying optimal screening strategies and approaches which take stakeholders preferences and perspectives into consideration and thus are likely to maximise screening uptake. The knowledge generated by this study will allow for development of recommendations to transform the current GDM screening programme to allow for sustained maximised rates of screening uptake.

\section{METHODS}

Three groups of participants are included in this research-patient participants, GP screening providers and hospital screening providers.
Patient participants were recruited from a larger sample who participated in the randomised controlled screening trial. ${ }^{16}$ Women were purposively sampled from this population to include those who had received a $75 \mathrm{~g}$ OGTT between 24 and 28 weeks gestation at either their GP, or at the hospital where they were receiving antenatal care, and women who tested positive and negative for GDM (in accordance with International Association of the Diabetes and Pregnancy Study Groups (IADPSG) criteria) at each location.

GP screening provider participants were recruited from a larger sample of GP providers who participated in the randomised controlled screening trial. ${ }^{16}$ GP screening providers were purposively sampled from this population to include those who (1) participated fully in the trial, (2) dropped out of the trial or (3) did not agree to participate in the trial.

Hospital screening providers were recruited from the three hospitals involved in the randomised controlled screening trial. ${ }^{16}$ Hospital screening providers were purposively sampled from this population to include nurses involved in scheduling, management and delivery of hospital screening services at all three hospital screening sites.

Telephone interviews were conducted with patient and GP screening provider participants, while in-person focus groups were conducted with the hospital screening provider participants in each of the three hospital sites where screening was conducted. Telephone interviews were utilised with the patient and GP screening provider participants as these were considered populations who are deemed hard to reach for face-to-face interviews. ${ }^{17}$ Telephone interviews are reported to be productive and successful in qualitative research. ${ }^{18}$

Semistructured interview guides were developed and utilised for each of the three participant groups. The interview guides included open-ended questions, to elicit as much information on the participants' experiences as possible. A conversational style of interviewing was adopted to encourage a comfortable and fluent dialogue which was rich in detail, while using the interview schedule as a reference to ensure that all key topics were covered. The research questions aimed to elicit the participants' perspectives of screening in each location including the benefits and barriers to screening at each location.

The interviews and focus groups were conducted by one of two of the authors (MT or AO'D), both postdoctoral researchers who had previous experience of conducting focus groups and interviews in clinical populations. The interviews and focus groups were audio-taped with the permission of each participant, using a digital recorder. Interviews were transcribed verbatim and analysed using theoretical thematic analysis with a semantic approach as outlined by Braun and Clarke. ${ }^{19}$ Thematic analysis is a particular type of qualitative analysis which focuses on recognising, analysing and reporting patterns (themes) within a qualitative data set. 
A semantic approach, focusing on the explicit meaning of the data, was chosen. This approach extends beyond a description of the themes to an interpretation of the significance and implications of the themes identified.

Transcripts were reviewed independently by two of the authors (MT and AO'D) who followed the phases outlined by Braun and Clarke. ${ }^{19}$ Phase 1 involved familiarisation with the data, reading and re-reading of the transcripts and noting of initial ideas. Phase 2 involved generating initial codes in a systematic fashion across the entire data set. Phase 3 involved collating the codes into potential themes. Phase 4 involved reviewing of the themes and ensuring that they are relevant in relation to both the coded extracts and the entire data set. Phase 5 involved refining the themes to ensure that they provide a clear reflection of the overall story portrayed in the data set. Both authors met regularly to identify common themes and discuss areas of agreement and divergence.

This study was conducted in accordance with the Declaration of Helsinki. An information letter was sent to each participant and informed consent was provided prior to interview or focus group participation.

\section{RESULTS}

This study includes 14 patient participants, 13 GP screening providers and 11 hospital screening providers.

Thematic analysis revealed four themes which are discussed in more detail below. Three are related to the benefits and barriers to screening at both assessed locations, while the fourth theme involves opinions on the optimal screening strategies.

\section{Theme 1: travel distance}

There appeared to be differences in opinions about the impact of travel distance on screening uptake depending on how far from the hospital screening site the woman was located. For women living in locations situated a long distance from the hospital setting, the provision of screening at their GP surgery which negated the requirement for an extensive journey was overwhelmingly viewed as a particularly advantageous. The necessity for extended fasting as part of the screening process reinforced the benefits of localised screening.

I do think the offering of it (GDM screening) through the GP was a very good idea especially as I am an hour away from my local hospital so I would have to go early in the morning and spend 3 and a half hours in the hospital. It's a long day when you haven't had anything to eat and you're sitting around waiting. Whereas because I did it at my GP you are local to home so when you are finished you are going home and you have something to eat fairly quickly. (Patient screened at GP)

This view was also shared by both GP and hospital providers. GP providers make reference to the additional stress that the long early morning journey causes.
I think it (screening at the GPs) is a lot easier than the hospitals. I think it (hospital screening) is a lot more hassle. Maybe if you're living in the city it is fine, but for someone living out here $(80 \mathrm{kms}$ from hospital), you're leaving here at half seven at the latest. (GP provider)

Noteworthy, hospital providers felt that currently their attendance rates for GDM screening are impacted by the distance the woman has to travel with those located furthest from the hospital setting less likely to attend.

\section{There is a role for them (GPs) in conducting OGTTs for women who live a distance from the hospital, in particu- lar for areas like Clifden $(75 \mathrm{kms})$ and Ennis $(65 \mathrm{~km})$ as these are locations where women are less likely to comply with OGTT referral and don't show. (Hospital provider)}

However, those women who lived equidistance from their GP and the hospital setting reported no preference for either location or in some cases preferred the hospital setting over the GP setting as a location to receive their screening.

It really doesn't bother me...it was the same thing for me to go to the hospital as it was for my own GP. (Patient screened in Hospital)

For the likes of me who are the same distance (from both locations) I would say it is the hospital (that I would prefer). (Patient screened at GP)

\section{Theme 2: best care provision}

Overall, the hospital setting was perceived to be superior to the GP setting for GDM screening in terms of perception of best care provision. Thematic analysis revealed four areas where differences are perceived between the GP and hospital setting namely provider expertise, specialised equipment, timeliness of results and standardisation of screening.

\section{Provider expertise}

From the patient perspective, GP screening providers were noted to show uncertainty about the procedures associated with completing the test which negatively influenced patients' perceptions of GP expertise in providing screening.

She (the GP) wasn't a hundred percent confident to be honest. She kind of kept looking at the instructions; she said 'this is all new'. In the hospital it just seems to flow, they were experts at it. (Patient screened at GP)

However, the GP screening providers did not report that the provision of the test was challenging to them indicating a discrepancy between the two participant groups.

It's pretty standard; my understanding is it is fairly reasonable. (GP provider) 
Patients appear to believe that the providers' ability to answer queries related to their pregnancy, GDM and screening is an important component of the screening process. Patients are aware that GPs are not specialists in the area of GDM or indeed obstetrics or endocrinology, and thus, many women believed that their GP did not have sufficient knowledge of the condition of GDM or its management to answer queries they had at the time of screening.

I think that it would be better(to have screening) in the hospital as you have a lot more access to people that might answer any questions if you are concerned whereas the GP might not be able to answer as many. (Patient screened in Hospital)

\section{Safety}

Women appeared concerned that their health may be compromised if an adverse event related to the GDM screening procedure were to occur in the GP setting; this was not a concern in the hospital setting as it is perceived to have all of the specialised equipment and staff to safely deal with such situations. Women perceive their GP as a generalised rather than acute care provider, less experienced in dealing with emergency situations. GP providers are also conscious of their potential inability to safely provide the screening.

I think the hospital might be better because GPs don't have as much equipment. When you take that liquid that has sugar in it (Polycal) you might get dizzy so anything can happen. So I think the hospital would be preferable. (Patient screened in Hospital)

In the ideal world the service should be offered locally but only if the facilities are available for it to be done safely. (GP provider)

\section{Timeliness of results}

Women also expressed concerns about how quickly the results of the screen would be relayed to them. Many women felt that there would be a longer delay in having results communicated to them if they were screened at the GP, and thus it would take longer to instigate GDM treatment where necessary. If screening occurred in the hospital setting, it was felt that results would be reported in a more expedited manner.

The problem with the GP would be that I would presume it takes longer for the results. (Patient screened in Hospital)

\section{Accuracy}

Despite the GP providers reporting their ability to conduct the test accurately as part of this trial, GP providers reported concern regarding the ability to maintain accurate standards in the long term, particularly in relation to the requirement for hourly blood draws. Many GPs believed that maintaining accuracy of the timing of blood draws would be difficult in the long term, considering the pressures on GP services in Ireland currently.

If you were saying that every antenatal lady were to have it (GDM screening) and it were to be accurate I'd have to say hand on heart that would be almost impossible to do. (GP provider)

Hospital providers also had concerns over the accuracy of GP-administered tests. Some hospital providers reported that on a number of occasions, GP provider standards during the trial were unsatisfactory.

They (GPs) are probably not used to doing glucose tolerance tests and for the odd one we discovered they might only have done a fasting and a two hour maybe. The test would be incomplete. (Hospital provider)

\section{Theme 3: sense of ease created}

Being in a space which promotes a sense of relaxation and comfort appears to be an important consideration for women in relation to the location that GDM screening is offered. In this cohort, women generally favoured the GP surgery as a location which offers higher levels of comfort during the screening appointment

The wait doesn't feel as long (and is) more comfortable at the GP. (Patient screened at GP)

It (hospital waiting area) is not a very easy area to relax in. It's not comfortable. I wouldn't really think that that area was a great area to wait in. (Patient screened in Hospital)

Furthermore, the hospital screening providers were cognisant of the limitations of the hospital setting in terms of comfort

There are disadvantages with having an OGTT in the hospital setting...overcrowded hot waiting room, sometimes with standing room only. (Hospital provider)

Furthermore, parking availability at the hospital setting and associated cost were also cited as disadvantages of the hospital settings which were mitigated in the GP setting. Both the women and the providers expressed that the lower stress related to parking at the GP setting added to the sense of ease created by this location.

More convenient in terms of parking and everything. (Patient screened in Hospital)

They don't have the added problems of things like parking fees. (GP provider)

\section{Theme 4: optimal screening}

The stakeholders involved in the GDM screening trial voiced their opinions about the optimum screening approach which they believed would lead to maximised uptake rates. Thematic analysis revealed three themes: 
(1) population screened, (2) screening provider and (3) screening location.

\section{Population}

Providers and patients differed in their views about to whom GDM screening should be provided. Generally, both GP and hospital providers believe that selective screening based on GDM risk factors is the most appropriate screening strategy. Economic and resource factors were cited as reasons for this decision.

It (universal screening) is a huge cost for probably some very low risk women. I mean there are a lot of young healthy women that you really don't think are high risk of diabetes and don't turn out to have it (GDM). I think defined criteria for at risk people (is preferable) rather than screening everybody. (GP provider)

However, the patients are more likely to be advocates for universal screening, indicating that they would like to have the offer of having screening conducted in all cases.

I think that everybody should probably be screened in an ideal world...I think it's important that as many women as possible would be screened. (Patient screened in Hospital)

\section{Provider}

All stakeholder groups agreed that the provider of GDM screening should have specialised knowledge and expertise in the area of GDM screening and management. This relates to the 'expertise' theme previously outlined where many women and hospital providers believed that GP providers lacked the expertise to provide information about GDM to their patients.

I actually feel it should be midwifery led. I'm not a midwife and there was times they'd ask me questions and I couldn't answer them. (Hospital provider)

A benefit of the hospital is that there is a lot more access to people that might answer questions, where the GP might not be able to. (Patient screened in Hospital)

\section{Location}

The three stakeholder groups were in agreement that a community location would be superior to the current hospital setting of the trialled GP setting for the provision of GDM screening. It was felt that a specialised service offered in the community setting such as in community health centres would incorporate all the benefits of the GP setting (ie, shorter travel distance, parking, comfort) and allow for the high standards of care associated with the hospital setting be maintained.

The ideal situation is organising it in the community health centres. (GP provider)

Definitely out in the community. (Hospital provider)

\section{DISCUSSION}

The aim of this study is to understand the perspectives of stakeholders about GDM screening in GP and hospital settings. Currently, neither location appears satisfactory at ensuring maximised screening uptake rates. ${ }^{2} 16$ Based on factors identified by stakeholders, we aim to make recommendations for a screening strategy for GDM that will potentially lead to improved uptake rates and therefore improved identification and management of the condition.

Cullinan et $\mathrm{al}^{14}$ recommended that reducing the distance that women had to travel to receive their screening would improve uptake rates. Thus, it is unsurprising that travel distance emerged as a theme in discussions around the provision of screening in the two settings. Thematic analysis indicates for women that live a considerable distance from the hospital setting, travel distance to the screening site is an important determinant of willingness to attend for GDM screening. Shorter travel distance emerged as the principal benefit of the GP screening setting over the hospital setting for these women as this factor includes not only travel time and cost but also the requirement for prolonged fasting associated with longer travel distance. GP and hospital providers also recognise long travel distances as barriers to screening in the hospital setting. For women who live equidistant from both settings, factors other than journey distance influence stakeholders' perspectives of the GP and hospital settings as screening locations.

One such factor was the perception of better care provision at the hospital setting. Women in this trial were aware that GPs are not specialists in the area of GDM and are less knowledgeable about management options and less able to manage an adverse event safely should it arise during the screening process. This perception of better care provision in the hospital setting has previously been reported in other care situations, for example, cancer survivors who cited the 'non-expert in cancer' nature of GPs as a main reason for not using GP services. ${ }^{20}$ The perception of GPs' lack of expertise and non-specialism in the area is likely to negatively influence patients' confidence in GPs' abilities to conduct the test in the appropriate manner and resultantly, the uptake of screening in this location. Furthermore, women were of the opinion that their screen results would take longer to process if the screen was conducted in the GP setting, and therefore it would take longer to initiate treatment where necessary. Perceived delay to treatment associated with GP screening is likely to impact womens' engagement with the GP setting as a location for the provision of GDM screening. Furthermore, the reality is that with part-time GP working, next day delivery of results and the communication of results via letter in many cases, means that the GP setting may significantly have to make changes to their delivery of care in order to successfully offer highquality GDM screening. Overall, the perception of better care provision in the hospital setting represents a 
significant real barrier to successfully utilising the GP setting to maximise uptake rates of GDM screening.

The GP setting was generally reported as the preferential location for GDM screening when considering the waiting period during the screening appointment. This is largely related to the comfort of the waiting area which, in the hospital setting, was off-putting to some women. This is particularly relevant as the waiting period for this test is quite long (approximately $2.5 \mathrm{~h}$ ). Arneill and Devlin ${ }^{21}$ found that the comfort of the waiting area was correlated with patients perception of quality of care; thus, it is likely that women in this study felt that the GP setting was advantageous in this regard. Parking, in terms of finding sufficient spaces as well as the financial cost, was also noted to be problematic in the hospital setting but not evident generally in the GP setting. Parking has frequently been mentioned as an attribute of patient satisfaction with primary care over secondary care ${ }^{22}$ and has commonly been included in taxonomies of patient satisfaction with care. ${ }^{2324}$ Thus, the sense of ease which is created by the GP setting but not by the hospital setting is an advantage of this location which can positively impact on uptake rates for GDM screening.

Despite attempting to impact the quantity of uptake, it must be ensured that in whatever location screening is provided, the quality of the test does not suffer. The accuracy of the test in relation to receiving three separate blood tests and receiving each test at hourly intervals was not deemed problematic by the patients in this trial at either of the locations. However, both the GP and hospital providers did feel that both of these aspects caused difficulties in the GP setting and could be problematic if there was a longer, larger roll out of such a programme in the GP setting. If the GP setting was to be utilised as a location for the provision of GDM screening, it would be of vital importance that GPs would be provided with additional training in order to provide an efficient and effective service, where high-quality accurate standards can be achieved routinely. If, after additional training and reorientation of service, high-quality and accurate standards cannot be maintained at the GP setting, it would appear unlikely that such a service could be recommended. It would also be of vital importance that both GPs and patients are confident that patient safety, particularly in the case of an adverse event, can be maintained in the GP setting. Similar to the necessity for accurate standards, if this aspect cannot be achieved in the GP setting, after additional training for providers in this clinical area, then it would be unlikely a GDM screening service in this location could be recommended. If a community location, as was suggested by stakeholders, is utilised, it is imperative that high standards, which equal or surpass the current hospital standards, are maintained irrespective of quantity, in order to appropriately treat GDM and avoid and minimise the related consequences.
This study benefitted from the inclusion of all of the key stakeholders involved in screening for GDM as part of the randomised controlled screening trial. Furthermore, our sampling strategy allowed inclusion of a wide variety of perspectives to be included, including women who received screening in both settings, with both positive and negative results for GDM, GPs who participated fully, in part and not at all in the randomised controlled screening trial and nursing staff from the three hospital sites of the randomised controlled screening trial. This allows us to be confident that the results reported are reflective of the key stakeholders. A limitation of the study is that the participants of this study were recruited from a defined regional area in Ireland, and thus the results may not be suitable for extrapolation to other areas nationally or internationally.

As previously outlined, the influence of travel distance appears to be only relevant for women living a considerable distance from the hospital setting, something not considered by the Cullinan et $a l^{14}$ study. Future research should assess at what distance does this become a factor and also consider the employment location of the women and the distance from this employment location to the screening site, which may be more important as a factor for women who are employed outside the home. Furthermore, in future GDM screening trials, taking into account the perspectives of the stakeholders identified as part of this qualitative study is prudent. It is very important to identify as many cases of GDM as possible through screening, to minimise the impacts GDM has on both maternal and neonatal health. Neither the hospital $^{2}$ nor the $\mathrm{GP}^{16}$ setting appears to provide the most appropriate set of factors to maximise uptake rates to allow for maximised identification of GDM cases. The data provided by stakeholders in this study can positively add to the findings of Cullinan et $a l^{14}$ and makes some further recommendations as to the optimal screening strategy for GDM screening which can maximise rates of uptake and thus minimise or avoid the short-term and long-term complications of the condition. It appears that a service, which is specialist led, provided conveniently local to the woman with good parking and comfort levels, provides accurate tests as well as advice and results in a manner which is deemed suitable to the women, is most suited in this population and, if put in place, should result in improved rates of screening uptake.

\section{Author affiliations}

${ }^{1}$ School of Medicine and Galway Diabetes Research Centre, National University of Ireland, Galway, Ireland

${ }^{2}$ School of Business and Economics, National University of Ireland, Galway, Ireland

${ }^{3}$ School of Psychology and Centre for Pain Research, National University of Ireland, Galway, Ireland

${ }^{4}$ Discipline of General Practice, National University of Ireland, Galway, Ireland

Contributors MT conducted focus groups and interviews, led qualitative analysis and drafted manuscript. AO conducted focus groups and interviews and assisted on qualitative analysis. AD assisted in acquisition of data. LC, 
BEM, LGG and FD were involved in conception of the study. All authors contributed to revising subsequent drafts and approved the final version.

Funding This work was supported by the Health Research Board Ireland grant number (ICE 2011/3).

Competing interests None declared.

Ethics approval Galway Regional Hospitals Research Ethics Committee.

Provenance and peer review Not commissioned; externally peer reviewed.

Data sharing statement Anonymised transcripts form part of the National University of Ireland, Galway archive, and can be made available to other bona fide researchers on request.

Open Access This is an Open Access article distributed in accordance with the Creative Commons Attribution Non Commercial (CC BY-NC 4.0) license, which permits others to distribute, remix, adapt, build upon this work noncommercially, and license their derivative works on different terms, provided the original work is properly cited and the use is non-commercial. See: http:// creativecommons.org/licenses/by-nc/4.0/

\section{REFERENCES}

1. American Diabetes Association. Diagnosis and classification of diabetes mellitus. Diabetes Care 2013;36(Suppl 1):S67-74.

2. O'Sullivan E, Avalos G, O'Reilly M, et al. Atlantic Diabetes in Pregnancy (DIP): the prevalence and outcomes of gestational diabetes mellitus using new diagnostic criteria. Diabetologia 2011:54:1670-5.

3. Hunt KJ, Schuller KL. The increasing prevalence of diabetes in pregnancy. Obstet Gynecol Clin North Am 2007;34:173-99, vii.

4. Dabelea D, Snell-Bergeon JK, Hartsfield CL, et al. Increasing prevalence of gestational diabetes mellitus (GDM) over time and by birth cohort Kaiser Permanente of Colorado GDM screening program. Diabetes Care 2005;28:579-84.

5. Catalano PM, McIntyre HD, Cruickshank JK, et al. The hyperglycemia and adverse pregnancy outcome study: associations of GDM and obesity with pregnancy outcomes. Diabetes Care 2012;35:780-6.

6. Schmidt MI, Duncan BB, Reichelt AJ, et al. Gestational diabetes mellitus diagnosed with a 2-h 75-g oral glucose tolerance test and adverse pregnancy outcomes. Diabetes Care 2001;24:1151-5.

7. Langer O, Yogev Y, Most O, et al. Gestational diabetes: the consequences of not treating. Am J Obstet Gynecol 2005;192:989-97.
8. Engeland A, Bjørge T, Daltveit AK, et al. Risk of diabetes after gestational diabetes and preeclampsia. A registry-based study of 230,000 women in Norway. Eur J Epidemiol 2011;26:157-63.

9. O'Sullivan JB. Diabetes mellitus after GDM. Diabetes 1991;40(Suppl 2):131-5.

10. Damm P. Future risk of diabetes in mother and child after gestationa diabetes mellitus. Int J Gynaecol Obstet 2009;104(Suppl 1):S25-6.

11. Kim C, Newton KM, Knopp RH. Gestational diabetes and the incidence of type 2 diabetes: a systematic review. Diabetes Care 2002;25:1862-8.

12. Noctor E, Crowe C, Carmody LA, et al. ATLANTIC-DIP: prevalence of metabolic syndrome and insulin resistance in women with previous gestational diabetes mellitus by International Association of Diabetes in Pregnancy Study Groups criteria. Acta Diabetol 2015;52:153-60.

13. Gayet-Ageron A, Poncet B, Guerre $\mathrm{P}$, et al. Specific information about the WHO guidelines for gestational diabetes screening improves clinical practices. J Eval Clin Pract 2008;14:36-42.

14. Cullinan J, Gillespie P, Owens L, et al. Accessibility and screening uptake rates for gestational diabetes mellitus in Ireland. Health Place 2012;18:339-48.

15. Agarwal MM. Gestational diabetes mellitus: an update on the current international diagnostic criteria. World J Diabetes 2015;6:782.

16. Tierney M, O'Dea A, Danyliv A, et al. Feasibility, acceptability and uptake rates of gestational diabetes mellitus screening in primary care vs secondary care: findings from a randomised controlled mixed methods trial. Diabetologia 2015;58:2486-93.

17. Opdenakker R. Advantages and disadvantages of four interview techniques in qualitative research. Qual Soc Res 2006;7:11.

18. Sturges JE, Hanrahan KJ. Comparing telephone and face-to-face qualitative interviewing: a research note. Qual Res 2004;4: 107-18.

19. Braun V, Clarke V. Using thematic analysis in psychology. Qual Res Psychol 2006;3:77-101.

20. Khan NF, Evans J, Rose PW. A qualitative study of unmet needs and interactions with primary care among cancer survivors. $\mathrm{Br} J$ Cancer 2011;105(Suppl 1):S46-51.

21. Arneill $A B$, Devlin AS. Perceived quality of care: the influence of the waiting room environment. J Environ Psychol 2002;22:345-60.

22. Concato J, Feinstein AR. Asking patients what they like: overlooked attributes of patient satisfaction with primary care. Am J Med 1997;102:399-406.

23. Howell EA, Concato J. Obstetric patient satisfaction: asking patients what they like. Am J Obstet Gynecol 2004;190:175-82.

24. Wuerth DB, Finkelstein SH, Kliger AS, et al. Patient assessment of quality of care in a chronic peritoneal dialysis facility. Am J Kidney Dis 2000;35:638-43. 